\title{
Relativistic Effects on Line Strengths for Transitions in the Hydrogenic Isoelectronic Sequence*
}

\author{
S. M. Younger** and A. W. Weiss \\ Institute for Basic Standards, National Bureau of Standards, Washington, D.C. 20234
}

(July 3, 1975)

\begin{abstract}
Relativistic line strengths have been computed for a large number of transitions using Dirac wave functions for the one-electron, hydrogen-like ions. As expected, the results indicate that relativistic effects are quite small for low stages of ionization. However, in general, they also remain small throughout a large portion of the isoelectronic sequence, becoming typically of the order of 10 percent in the vicinity of $Z=50$, after which they grow quite rapidly. This suggests that for multielectron ions a basically nonrelativistic theory might well be adequate for light atom isoelectronic ions through as much as 30 or 40 stages of ionization.

Key words: Dirac theory; hydrogen; line strength; oscillator strength; relativistic corrections; spectroscopy.
\end{abstract}

\section{Introduction}

The regular behavior of atomic oscillator strengths along isoelectronic sequences has proved to be a useful tool for analyzing a large body of $f$-value data $\lceil 1\rceil .{ }^{1}$ The graphical analysis of such data has also made possible the prediction, by interpolation, of much previously unknown data for ionized species [2].

The theoretical underpinning of such analyses is the charge expansion theory [3] which predicts that the large $Z$ limit for a multiplet strength is essentially given by the hydrogenic case. If there is no large $Z$ degeneracy, e.g., the $2 s-2 p$ degeneracy, then the limiting value is the hydrogenic strength multiplied by a manyelectron angular factor appropriate to the particular $L S$-states involved. If there is an asymptotic degeneracy, then one must coherently mix the square roots of the line strengths by a simple first order energy diagonalization; the radial integrals, however, are still hydrogenic.

This scheme is based on a perturbation expansion of the nonrelativistic, many-electron Schrödinger equation in inverse powers of the nuclear charge. The theory thus encompasses an entire isoelectronic sequence at a time. For large nuclear charge, however, it is natural to expect that relativistic corrections will

* Supported by Energy Research and Development Administration.

** Permanent address: University of Marvland, College Park, Md, 20740.

' Figures in brackets indicate the literature reterences. become important, so that eventually these isoelectronic sequence analyses must break down. If one is interested in predictions for highly ionized species, then it is important to determine at what stage of ionization and in what way relativistic effects begin to make themselves felt.

The most obvious effect, and probably the first in importance, is the traditional intermediate coupling [4]. In this scheme multielectron states of different $L$ and $S$ are coupled together by the off-diagonal spinorbit interaction, as obtained from a Pauli-approximation reduction of the Dirac Hamiltonian. The wave function is still a linear combination of nonrelativistic functions, and the line strengths are affected by a coherent mixing of the nonrelativistic transition moments.

In addition to this, however, relativistic effects also modify the orbitals themselves, thus more directly affecting the line strengths. To get some idea of the magnitude of this orbital effect for highly ionized systems, we have therefore computed the hydrogen-like line strengths according to Dirac theory for a large number of transitions, following them along the entire isoelectronic sequence through $Z=100$, and comparing with the non-relativistic predictions.

While a number of authors have discussed the Dirac line strengths for large $\mathrm{Z}$ ions, they seem to have concentrated on the $1 s-n p$ resonance lines, primarily in order to approximate the $K$-shell x-ray intensities [5-8]. Our orientation here is somewhat different. 
We would like to look on these calculations as a kind of prototype of the relativistic orbital effects one might find in highly ionized multielectron species. Thus, e.g., the $2 s-2 p$ line strengths are calculated with an eye to the resonance lines of the lithium isoelectronic sequence. While we are primarily interested in the qualitative questions of the manner and rate of growth of relativistic effects along the sequence, our results may prove useful as approximate correction factors for some nonhydrogenic ions.

\section{Formulas and Details of the Calculation}

We calculated the dipole line strengths as a function of $\mathrm{Z}$ using the hydrogen-like Dirac wave functions. In evaluating the matrix elements, we followed the analysis of Babushkin [8], as recently reviewed by Garstang [9]. For a detailed discussion, the reader is referred to Garstang's review; we quote here only the pertinent formulas.

For the case where variation of the electromagnetic field over atomic dimensions is included, the electric dipole line strength for $l \rightarrow l+1$ is given by:

$$
\begin{aligned}
& S=\frac{9 l(l+1)}{2(2 l+1)} \frac{1}{k^{2}} I^{2} \\
& \text { for } j=l-1 / 2 \rightarrow j^{\prime}=l+1 / 2, \\
& S=\frac{9(l+1)}{2(2 l+1)(2 l+3)} \frac{1}{k^{2}} I^{2} \\
& \text { for } j=l+1 / 2 \rightarrow j^{\prime}=l+1 / 2, \\
& S=\frac{9(l+1)(l+2)}{2(2 l+3)} \frac{1}{k^{2}} I^{2} \\
& \text { for } j=l+1 / 2 \rightarrow j^{\prime}=l+3 / 2,
\end{aligned}
$$

where $I=\left[R_{1}+R_{2}+R_{3}-R_{4}+\left(\kappa_{1}-\kappa_{2}\right)\left(R_{3}+R_{4}\right)\right]$, and

where

$$
\begin{aligned}
R_{1} & =\int_{0}^{\infty} g_{1} g_{2} r^{2} j_{L}(k r) d r \\
R_{2} & =\int_{0}^{\infty} f_{1} f_{2} r^{2} j_{L}(k r) d r \\
R_{3} & =\int_{0}^{\infty} f_{1} g_{2} r^{2} j_{L-1}(k r) d r \\
R_{4} & =\int_{0}^{\infty} f_{2} g_{1} r^{2} j_{L-1}(k r) d r \\
k & =\frac{2 \pi}{\lambda},
\end{aligned}
$$

with $L=1 . f$ and $g$ are the small and large components, respectively, of the Dirac spinor,

$$
\psi=\left(\begin{array}{c}
g(r) \chi_{\kappa} \eta \\
-i f(r) \chi_{-\kappa, \eta}
\end{array}\right),
$$

and the subscripts differentiate between the initial and final states. A discussion of these wave functions is given by Bethe and Salpeter [10]. The Dirac quantum number $\kappa$ is defined as:

$$
\kappa=\begin{array}{r}
-(l+1) \text { if } j=l+1 / 2 \\
l \text { if } j=l-1 / 2 .
\end{array}
$$

If one neglects the variation of the field over the dimensions of the atom, which was found to be a reasoriable approximation, the expressions for the corresponding $j \rightarrow j^{\prime}$ transitions are:

$$
\begin{aligned}
& S=\frac{2 l(l+1)}{2 l+1} I^{2}, \\
& S=\frac{2(l+1)}{(2 l+1)(2 l+3)} I^{2}, \\
& S=\frac{2(l+1)(l+2)}{2 l+3} I^{2},
\end{aligned}
$$

where

$$
I=\int_{0}^{\infty} r^{3}\left(f_{1} f_{2}+g_{1} g_{2}\right) d r
$$

The integral transformation to equivalent forms involving products of large and small components in the integrand was not used because of numerical difficulties for low and intermediate values of $Z$. The above form is also more useful for sorting out specific relativistic effects, since it displays separately the large and small component contributions to the nonretarded integrals. The integrals were evaluated both numerically and analytically - the latter using term by term integration of the radial functions. Doing the integrals both ways provided a mutual check on the calculations as well as providing detailed information on such effects as cancellations in the integrand due to relativistic orbital shifts, etc.

\section{Results and Discussion}

As mentioned earlier, the $1 s-n p$ series has already been dealt with by Payne and Levinger [6] and Babushkin [8], among others. While calculations were done on a large number of transitions and ions, we present results here only for the $2 s-2 p, 2 s-3 p$, $2 p-3 d, 2 p-3 s, 3 s-3 p$, and $3 p-3 d$ transitions as typical of the relativistic behavior of these hydrogenic line strengths. Furthermore, these are prototypes of the radial integrals needed for resonance transitions in ions isoelectronic with first and second row atoms. The ratios of the relativistic 
to nonrelativistic line strengths are shown graphically in figures $1-4$ for the $s-p$ transitions. $Z^{2} \mathrm{~S}$, rather than the line strength itself, is the natural quantity of interest since the nonrelativistic value is a constant, independent of Z. Deviations from this constant thus display directly the effects of relativity on the line strength.

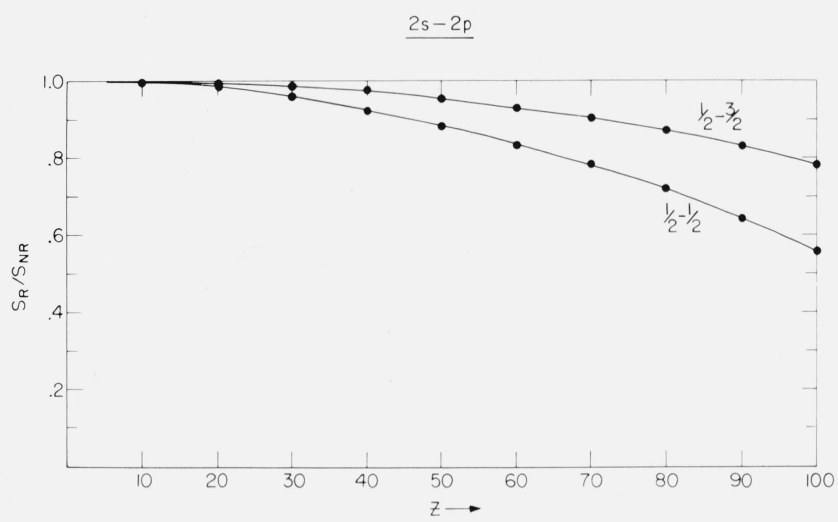

FiguRE 1. Ratio of relativistic to nonrelativistic line strengths versus nuclear charge for the $2 \mathrm{~s}-2 \mathrm{p}$ transitions in the hydrogen isoelectronic sequence.

The nonrelativistic values of $Z^{2} S$ are 18.0 and 36.0 for the $1 / 2-1 / 2$ and $1 / 2-3 / 2$ lines respectively.

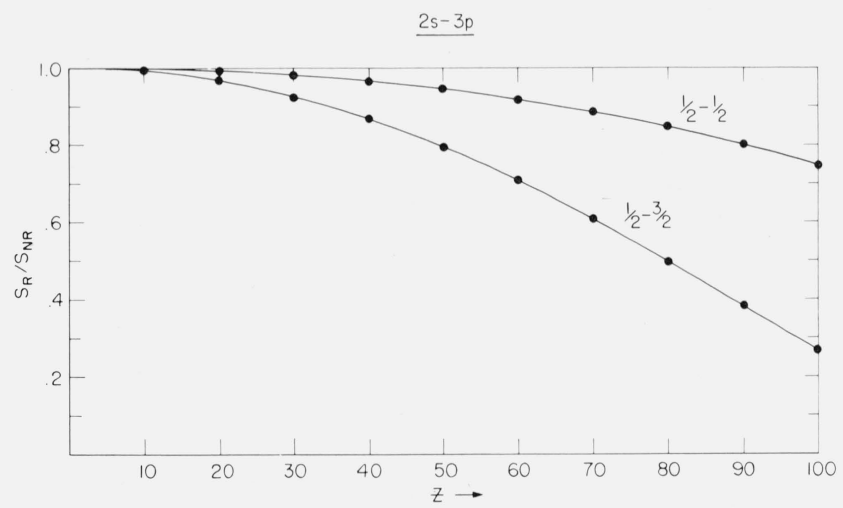

Figure 2. Ratio of relativistic to nonrelativistic line strengths versus nuclear charge for the $2 \mathrm{~s}-3 \mathrm{p}$ transitions in the hydrogen isoelectronic sequence.

The nonrelativistic values of $Z^{2} S$ are 6.26 and 12.52 for the $1 / 2-1 / 2$ and $1 / 2-3 / 2$ lines respectivel\%.

Usually one finds the relativistic line strength to decrease with increasing $\mathrm{Z}$, reflecting the well-known contraction of the relativistic wave function toward the origin. As the electron distribution is drawn in toward the nucleus, greater emphasis is placed on smaller radii in the transition integrand. Furthermore, the normal behavior is for the large component contribution to decrease, while that of the small component increases. However, even at large $\mathrm{Z}$ the small component term is still only a few percent of the larger, the overall behavior thus being dominated by the large component.

An example of departure from this behavior occurs in the $2 p-3 s$ transition. While the $1 / 2-1 / 2$ transition

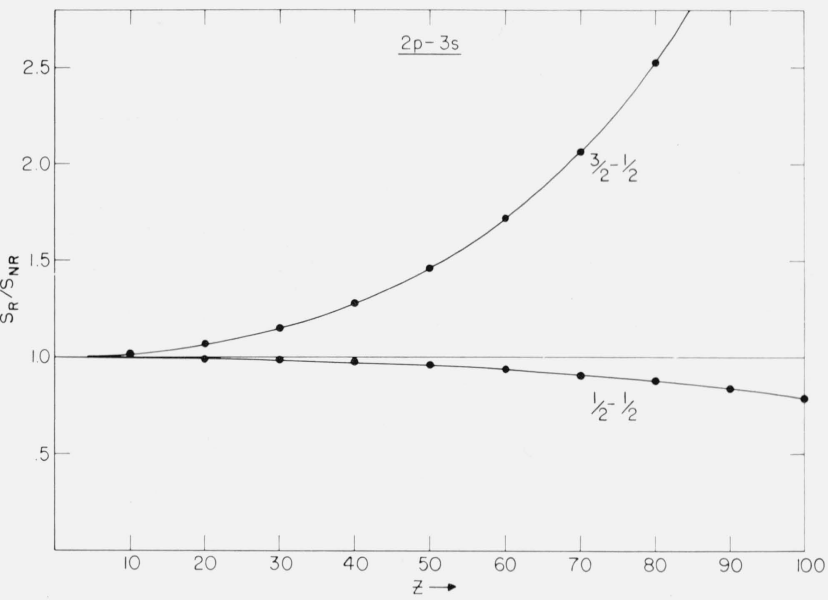

Figure 3. Ratio of relativistic to nonrelativistic line strengths versus nuclear charge for the $3 \mathrm{~s}-2 \mathrm{p}$ transitions in the hydrogen isoelectronic sequence.

The nonrelativistic values of $Z^{2} S$ are 0.587 and 1.174 for the $1 / 2-1 / 2$ and $1 / 2-3 / 2$ lines respectively.

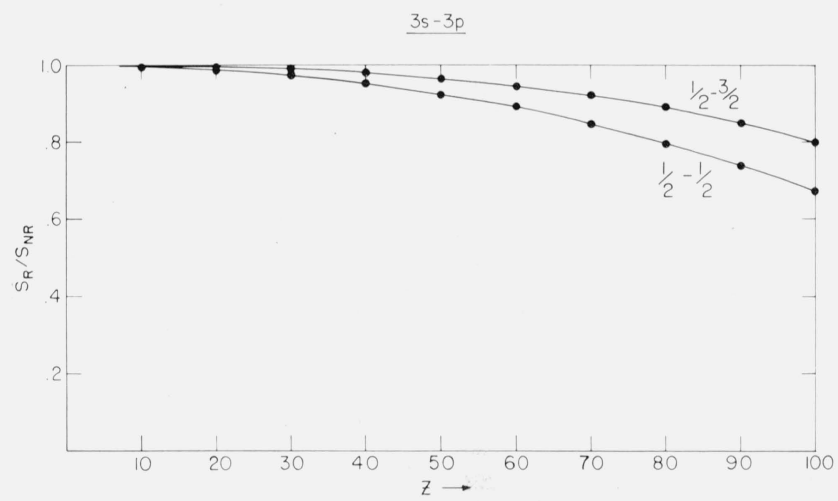

FIGURE 4. Ratio of relativistic to nonrelativistic line strengths versus nuclear charge for the $3 \mathrm{~s}-3 \mathrm{p}$ transitions in the hydrogen isoelectronic sequence.

The nonrelativistic values of $Z^{2} S$ are 108.0 and 216.0 for the $1 / 2-1 / 2$ and $1 / 2-3 / 2$ lines respectively.

follows the usual pattern of decreasing strength with increasing $Z$, the $3 / 2-1 / 2$ transition grows larger than the nonrelativistic value as $\mathrm{Z}$ increases. The explanation appears to be related to differential interference effects in the transition integral for the two transitions. There is substantial cancellation in the $2 p-3 s$ integral for the nonrelativistic case-the positive-negative ratios of the integrand being about $2: 1$. As $\mathrm{Z}$ increases, the $2 p_{1 / 2}$ and $3 s_{1 / 2}$ orbitals contract by roughly the same amount maintaining approximately the same integrand ratio. The $2 p_{3 / 2}$, however, does not contract nearly as rapidly, resulting in a significant increase in the integrand ratio, i.e., less cancellation and so a larger transition integral.

The most important observation about these calculations, however, concerns the rate of growth of the relativistic corrections along the isoelectronic sequence. For sufficiently large $Z$, the relativistic line strength "takes off" and ultimately departs quite drastically from the nonrelativistic one. These cor- 
rections, however, don't seem to take hold until the nuclear charge becomes rather large. Thus, for $Z=50$, which is forty-nine times ionized tin, the relativistic line strengths rarely deviate by more than 10 percent from the nonrelativistic. An exception to this is the $2 p_{3 / 2}-3 s_{1 / 2}$ transition discussed above, which deviates by about 30 percent.

This slow increase in the relativistic effect is further illustrated in tables 1 and 2, where we present the actual numerical results for selected ions for some $p-d$, as well as $s-p$ transitions. In these tables the strengths and doublet ratios for $\mathrm{Z}=1$ are the nonrelativistic values so that the remaining numbers display directly the relativistic deviations. At $Z=50$, for example, one sees corrections ranging from 3 percent to 14 percent for both the doublet ratios as well as the absolute line strengths. Such general conclusions are consistent with the results of Burke and Grant [11], who calculated the Dirac hydrogenic expectation values of $r^{\mathrm{N}}$ and found small and slowly increasing relativistic corrections. Indeed, there is even fairly good semiquantitative agreement between our dipole transition moments and their results for $\mathrm{N}=1$.

TABLE 1. Relativistic line strengths and doublet ratios for $2 \mathrm{~s}-2 \mathrm{p}$ and $3 \mathrm{~s}-3 \mathrm{p}$ transitions in the hydrogen isoelectronic sequence

The $Z=1$ results are the nonrelativistic predictions.

\begin{tabular}{c|c|c|c|c|c|c}
\hline \hline \multirow{5}{*}{$2 s-2 p$} & \multicolumn{3}{|c|}{$1 / 2-1 / 2$} & \multicolumn{2}{c|}{$1 / 2-3 / 2$} & \multirow{2}{*}{$\begin{array}{c}\text { Doublet } \\
\text { ratio }\end{array}$} \\
\cline { 2 - 6 } & $\mathrm{Z}$ & $\mathrm{Z}^{2} \mathrm{~S}$ & $\mathrm{~S}_{\mathrm{R}} / \mathrm{S}_{\mathrm{NR}}$ & $\mathrm{Z}^{2} \mathrm{~S}$ & $\mathrm{~S}_{\mathrm{R}} / \mathrm{S}_{\mathrm{NR}}$ & \\
\cline { 2 - 6 } $35-3 p$ & 1 & 18.0 & 1.0 & 36.0 & 1.0 & 2.00 \\
& 10 & 17.92 & 0.996 & 35.94 & 0.998 & 2.01 \\
& 30 & 17.28 & .960 & 35.42 & .983 & 2.05 \\
& 50 & 16.01 & .890 & 34.33 & .953 & 2.14 \\
& 70 & 14.11 & .784 & 32.56 & .904 & 2.31 \\
& 90 & 11.57 & .643 & 29.85 & .829 & 2.58 \\
& 10 & 108.0 & 1.0 & 216.0 & 1.0 & 2.00 \\
& 30 & 104.9 & 0.997 & 215.7 & .999 & 2.00 \\
& 50 & 99.6 & .971 & 213.2 & .987 & 2.03 \\
& 70 & 91.2 & .922 & 207.8 & .962 & 2.09 \\
& 90 & 79.8 & .739 & 198.7 & .920 & 2.18 \\
& & & & 183.9 & .851 & 2.30 \\
\hline
\end{tabular}

While it is tempting to extend these conclusions to the more general case of a multielectron ion, one should do so with some care, and such considerations must remain somewhat conjectural. Probably the safest conclusion is the general qualitative one that the orbital relativistic corrections to a line strength generally tend to remain small, probably not greater than 10 percent up to fairly high $\mathrm{Z}$, say 30 or 40 . It would thus appear safe to take the nonrelativistic values for ions where no intermediate coupling effects are expected. For example, the resonance $3 s-3 p$ transitions of sodium-like ions are probably fairly well represented by a nonrelativistic calculation throughout this range of Z. For more accuracy or higher Z, one might even want to apply the correction factors obtained here, namely, the $S_{R} / S_{N R}$ ratios in tables 1 and 2, although at the present this possibility must remain a conjecture.

There are, however, several reservations which must be kept in mind. In the first place, if one has a transition with a substantial amount of cancellation, small changes in the orbitals can alter the delicate interference pattern in the transition integrand enough to lead to relatively large changes in the transition integral. In such cases a small orbital relativistic effect manifests itself in a much larger line strength correction; see, e.g., our results on the $2 p-3 s$ transitions. Secondly, one should remember that for any realistic one-electron model of the multielectron ion, such as the Hartree-Fock, the orbital nodal structure is not hydrogenic. The effective charge changes from the inner to outer parts of the wave function. For a sodiumlike ion, for example, the $1 s$ electron sees an effective nuclear charge of essentially $\mathrm{Z}$ while the $3 s$ electron sees a screened charge more like Z-10, and orthogonalization produces a $3 s$ orbital with a nonhydrogenic loop pattern, although this effect should tend to diminish for increasing $\mathrm{Z}$.

While the oscillator strength is often the most convenient quantity to extrapolate in an isoelectronic sequence, one should note that it will deviate much sooner from the nonrelativistic value because of large and rapidly increasing relativistic corrections to the transition energy. The oscillator strength is given by

TABLE 2. Relativistic line strengths and doublet ratios for the $2 \mathrm{p}-3 \mathrm{~d}$ and $3 \mathrm{p}-3 \mathrm{~d}$ transitions in the hydrogen isoelectronic sequence

The $Z=1$ results are the nonrelativistic predictions.

\begin{tabular}{|c|c|c|c|c|c|c|c|c|}
\hline & \multicolumn{3}{|c|}{$1 / 2-3 / 2$} & \multicolumn{2}{|c|}{$3 / 2-3 / 2$} & \multicolumn{2}{|c|}{$3 / 2-5 / 2$} & \multirow{2}{*}{$\begin{array}{c}\text { Doublet } \\
\text { ratios }\end{array}$} \\
\hline & $\mathrm{Z}$ & $\mathrm{Z}^{2} \mathrm{~S}$ & $\mathrm{~S}_{\mathrm{R}} / \mathrm{S}_{\mathrm{NR}}$ & $Z^{2} S$ & $\mathrm{~S}_{\mathrm{R}} / \mathrm{S}_{\mathrm{NR}}$ & $\mathrm{Z}^{2} \mathrm{~S}$ & $\mathrm{~S}_{\mathrm{R}} / \mathrm{S}_{\mathrm{NR}}$ & \\
\hline \multirow[t]{6}{*}{$2 p-3 d$} & 1 & 30.00 & 1.0 & 6.00 & 1.0 & 54.00 & 1.0 & 5.00:1:9.00 \\
\hline & 10 & 29.89 & 0.996 & 6.00 & 1.0 & 53.99 & 1.0 & 4.98:1:9.00 \\
\hline & 30 & 28.58 & .953 & 5.93 & 0.988 & 53.51 & 0.991 & 4.82:1:9.02 \\
\hline & 50 & 25.98 & .866 & 5.79 & .965 & 52.55 & .973 & $4.48: 1: 9.07$ \\
\hline & 70 & 22.11 & .737 & 5.59 & .932 & 51.11 & .946 & $3.96: 1: 9.15$ \\
\hline & 90 & 17.09 & .570 & 5.31 & .885 & 49.18 & .911 & $3.22: 1: 9.26$ \\
\hline \multirow[t]{6}{*}{$3 p-3 d$} & 1 & 135.0 & 1.0 & 27.0 & 1.0 & 243.0 & 1.0 & $5.00: 1: 9.00$ \\
\hline & 10 & 134.9 & 0.999 & 26.96 & 0.999 & 242.7 & 0.999 & $5.00: 1: 9.00$ \\
\hline & 30 & 134.0 & .993 & 26.66 & .987 & 241.7 & .995 & 5.03:1:9.07 \\
\hline & 50 & 132.2 & .979 & 26.05 & .965 & 239.6 & .986 & $5.07: 1: 9.20$ \\
\hline & 70 & 128.9 & .955 & 25.13 & .931 & 236.4 & .973 & $5.13: 1: 9.41$ \\
\hline & 90 & 123.2 & .913 & 23.93 & .886 & 232.1 & .956 & $5.15: 1: 9.70$ \\
\hline
\end{tabular}




$$
f=\frac{2}{3} \frac{\Delta \boldsymbol{E}}{g} S,
$$

where $g$ is the lower state degeneracy, $S$ the line strength, and $\Delta E$ the transition energy. Even if $S$ remains essentially nonrelativistic, a large first order relativistic correction to $\Delta E$ will have a large effect on $f$. The line strength itself would thus appear to be the safest and most natural quantity to extrapolate to large $\mathrm{Z}$ ions.

In conclusion it appears reasonable to suggest that the first and largest effect of relativity for a light atom isoelectronic sequence will be that due to intermediate coupling. Furthermore, one should be able to calculate these effects reasonably well (providing first order energy shifts are included) with a nonrelativistic basis through something like 30 or 40 stages of ionization. For ionic systems where intermediate coupling does not occur, a nonrelativistic calculation should give the line strengths with adequate accuracy through a similar range of ionization stages. Beyond an effective $Z$ of 50 , it appears that relativistic orbital effects will become sufficiently large to necessitate a more fully relativistic treatment.

\section{References}

[1] Wiese, W. L., and Weiss, A. W., Phys. Rev. 175, 50 (1968).

[2] Smith, M. W., and Wiese, W. L., Astrophys. J. Suppl. Ser. 23, \# 196, 103-192 (1971).

[3] Layzer, D., Ann. Phys. (New York) 8, 271 (1959).

[4] Condon, E. U., and Shortley, G. H., Theory of Atomic Spectra (Cambridge University Press, New York, 1955).

[5] Massey, H. S. W., and Burhop, E. H. S., Proc. R. Soc. A 153, 661 (1936).

[6] Payne, W. B., and Levinger, J. S., Phys. Rev. 101, 1020 (1956).

[7] Scofield, J. H., Phys. Rev. 179, 9 (1969).

[8] Babushkin, F. A., Opt. Spektrosk. 13, 141 (1962); Opt. Spektrosk. 19, 1 (1965); Acta Phys. Pol. 25, 749 (1964); Sov. Phys.-JETP 21, 372 (1965).

[9] Garstang, R. H., in Topics in Modern Physics-A Tribute to Edward U. Condon, Edited by W. E. Brittin and H. Odabasi, pp. 153-167 (Colorado Associated University Press, Boulder, Colorado, 1971).

10] Bethe, H. A., and Salpeter, E. E., Quantum Mechanics of One and Two Electron Atoms (Academic Press, New York, 1957).

[11] Burke, V. M., and Grant, E. I., Proc. Phys. Soc. 90, 297 (1967).

(Paper 79A5-864) 